\title{
Plaisir du texte et imaginaire numérique de la littérature, à partir de François Bon
}

\author{
Ana Paula Coutinho* \\ Université de Porto - ILCML
}

Résumé : Il n’y a jamais eu autant de livres publiés (y compris de littérature), mais le nombre de lecteurs est loin d'avoir augmenté dans la même proportion. L'invasion du numérique dans la vie contemporaine semble avoir dicté la fin du livre, du moins tel qu'on le connaît depuis Gutenberg, mais cela ne signifie point la fin ni de l'écriture ni de la lecture littéraires, plutôt leur mutation particulièrement accélérée. Le travail de François Bon, auteur, entre autres, des essais Après le livre (Seuil, 201I) et de La Société des Amis de L'Ancienne Littérature (2020), nés au sein de sa plateforme de réflexion et d'expérimentation www.tierslivre.net, constitue le noyau de mon article qui se propose de mettre en évidence et en relation les propositions de cet auteur polyédrique, car elles représentent les fondements-même d'une écriture-lecture créative à l'ère du numérique, aussi bien que leurs enjeux en termes de "plaisir du texte ", à fin de parvenir à une « renaissance » du livre conçu comme composition plurisensorielle d'un distancement élargissant du monde.

Mots-clés : François Bon, Livre, Plaisir du texte, Remédiation, Site

Abstract: There have never been so many books published (including literature), but the number of readers is far from increasing in the same proportion. Apparently, digital invasion in contemporary life imposed the end of the book, at least as we know it since Gutenberg, which does not necessarily mean the end of literary writing and reading, but rather their mutation, particularly accelerated. The work of François Bon, author, among others, of the Après le Livre (Seuil, 201I) and La Société des Amis de L'Ancienne Littérature (2020) essays, both born within his digital platform - www. tierslivre.net -, are the center of my article, which intends to highlight the proposals of this multimode author, because they represent the foundation of a linked literary writing-reading in digital age, as well theirs challenges to "the pleasure of the text », in order to a « rebirth » of the book as a multisensory composition of a widening distance from the world. 
Ana Paula Coutinho

Keywords: François Bon, Livre, Plaisir du texte, Remédiation, Site

Mais qu'on se saisisse de l'écran comme d'un monde en relief, quel livre inventons-nous a peine décelable encore?

François Bon

Le fait de présenter le travail de François Bon pour réfléchir aux enjeux de « lire et écrire des textes littéraires à l'époque des humanités numériques ", érigés en projet regroupant des chercheurs de différentes universités européennes' est loin de représenter un choix arbitraire, étant donné qu'il s'agit là d'un pionnier incontestable dans les rapports entre activité littéraire et plateforme informatique sur lesquels il se penche depuis presque un quart de siècle. En fait, c'est en 1997 que François Bon, à l'époque déjà auteur d'une dizaine de livres publiés et écrivain bien reconnu par la critique, a créé une première page personnelle qui s'avérerait bien plus qu'une expérience éphémère, plutôt l'embryon d'un engagement constant envers les enjeux du cyberespace et de la littérature contemporaine française (et autres).

Très vite devenu un catalyseur de dynamiques littéraires sur le web, et ayant pris l'habitude d'y réunir d'autres auteurs, le site de François Bon devait donner lieu, en 1999, à une revue littéraire, ${ }^{2}$ puis à une structure associative (2000), dont le nom, remue.net, aura voulu répondre implicitement à ceux qui se demandaient si la littérature française bougeait encore, à part l'hommage fait, bien-sûr, au recueil de poèmes - La nuit remue - d'Henri Michaux. Pourtant, et par ailleurs, « remue » fait penser aussi à la remue, c'est-à-dire à la transhumance, ce qu'on peut interpréter comme métaphore du passage temporaire, de l'alternance, entre différents territoires de créativité, ce qui rejoint une idée pratique partagée de littérature en tant qu' « acte et capacité de dire et d'inventer, récit du réel de ce monde dans lequel nous vivons et que nous avons en partage, espace de création littéraire en relation avec les autres arts et les formes de création que permet Internet.»- suivant les propositions avec lesquelles le collectif remue.net continue de se présenter. ${ }^{3}$

Quelques années après, en 2004, François Bon s'est éloigné de ce site pour mieux s'adonner aux dérivations de son travail d'écriture moyennant la fondation d'un autre site, personnel cette fois-ci au titre rabelaisien - Le Tiers Livre - qui, depuis, n'a cessé de s'élargir et de se créer une large arborescence. À l'évocation directe à Rabelais, François Bon a voulu ajouter l'idée « d'une présence tierce du livre, un livre à côté des livres » (Bon 2011 : 64), d'où l'arborescence du site tierslivre.net faite 
de multiples liens qui, de temps en temps, se réorganisent et se renouvellent pour s'adapter aux contraintes et à l'évolution de l'univers numérique.

Ce n'est certes pas un menu détail si ce Tiers Livre de François Bon compte, à cette date, environ 4 mille articles, s'il a diffusé depuis 2009 plus de 1500 vidéos, si sa chaîne Youtube a plus de 5500 abonnés et si, parallèlement, sa liste de livres publiés compte déjà une cinquantaine de titre... Entre outre, cela prouve que nous avons affaire à un cas extraordinaire d'hyperactivité créative, marquée par une écriture aussi débordante que résistante. En fait, la consistance, la permanence et le rayonnement de tierslivre.net ne peuvent, en aucun cas, être comparés à des ressources auxiliaires comme une page d'internet ou à un blog d'écrivain, ceux-ci étant conçus comme vitrines de promotion d'une œuvre littéraire faite en dehors du web. Cette distinction justifierait déjà, en elle-même, que l'on parle de la singularité du travail de François Bon dans le cyberespace. ${ }^{4}$ Mais il y a plus : sa prolixité et sa créative discursive utilisant aussi bien la parole, que l'image et le son, s'accompagnent depuis environ deux décennies et d'une réflexion sur les conséquences du numérique, ${ }^{5}$ et d'une grande auto-exigence technologique, ce qui fait du tierslivre.net un espace fondamental d'expérimentation et de partage d'écriture(s)-lecture(s) avec toujours les textualités du monde contemporain comme horizon, ce qui s'avère fort stimulant pour les questionnements autour des(s) plaisir(s) de lire de nos jours.

À ce propos, il me semble pertinent de commencer par souligner l'enthousiasme, fort interpelant, avec lequel François Bon se détourne de la jérémiade habituelle sur l'abandon ou la fin et du livre et de la lecture, comme s'il s'agissait de réalités tout à fait synonymes, et des formes non-historiques, donc immuables. Pour cet auteur, penser "l'après le livre ", comme il le fait ouvertement dans un essai homonyme publié en 20II, ne signifie nullement une quelconque « haine du livre », comme celle que l'on voit parfois se répandre par ignorance, par méfiance ou par indifférence, des attitudes communes aujourd'hui, comme, hélas !, dans le passé plus ou moins lointain. Au fil des pistes, fragments et incisives qui reprennent des réflexions partagées auparavant sur son site, François Bon insiste beaucoup sur ce que nous assistons « à un autre changement d'appareil de cette forme de technologie qu'a toujours été l'écriture » (Bon 2011 : 188), une mutation aussi paradigmatique que celle de l'invention de l'imprimerie au XVème siècle, mais qui ne signifie pas (ou ne doit pas signifier) la fin de l'écriture et de la lecture littéraires. Il n'empêche que les notions d'auteur et de livre ne sont pas séparables des conditions matérielles d'énonciation ou de reproductibilité (idem: 11 ), raison pour laquelle il est de plus en plus difficile, voire impossible, de concevoir aussi bien l'écriture que la lecture tout à fait en marge de notre époque envoûtée de cyberculture.

La proposition d'" un imaginaire numérique de la littérature " avancée aussi dans son dernier livre, Société des Amis de l'Ancienne Littérature (2020), à partir de fictions ou de chroniques préalablement écrites en ligne, lors de voyages de l'auteur 
aux EUA et au Québec, passe par la redéfinition des notions de livre et d'auteur, en articulation directe avec les pratiques et les fonctions de l'archivage, des images, du blog et du vlog, des bases de données et des liens.

François Bon est fort conscient de ce que, à moins de faire l'autruche, le livre ne peut plus être conçu aujourd'hui comme un seul «Assemblage de feuilles manuscrites ou imprimées destinées à être lues », pour reprendre la définition que l'on trouve dans le Dictionnaire de l'Académie Française. ${ }^{6}$ Même ceux qui continuent d'écrire à la main ou de lire des livres en papier, ou ne lisent que sur du papier, ne peuvent éviter que les travaux d'édition de texte et d'imprimerie soient maintenant faits par des logiciels, ou que la conception graphique des livres soit de plus en plus alignée sur l'ergonomie des sites web, même s'ils ne sont pas ce que l'on nomme des livres numériques, que François Bon voit seulement comme une «bulle transitoire » dans le contexte d' " un monde en bascule » (Bonnet 2012), dont cet auteur se laisse imprégner et transformer, comme un impératif kantien. Pour l'auteur d'Autobiographie des objets (2012), le grand défi sera, donc, de savoir comment reconduire, vers le numérique et vers le web, l'essentiel de l'énergie, du souffle, d'une forme d'écriture, comme la littéraire, qui a comme spécificité la figuration de l'instabilité, des passerelles, enfin, du tumulte du monde. Désormais, les idées de bouillonnement et de chaos, aussi bien du monde social que du mode d'énonciation, seront toujours à la base d'une nouvelle forme d'écriture automatique, non préméditée, que l'auteur poursuit devant l'écran de son ordinateur, mais souvent en articulation directe avec d'autres performativités.

Le livre de fictions Tumulte, publié en 2006 chez Fayard, résultait d'une année de publications ou de "performance web », d'après ses propres mots, faites directement sur serveur internet, du I"r mai 2005 au II mai 2006. Or, si, d'un côté, nous pouvons suivre le raisonnement de certaines analyses, qui voient dans la démarche de François Bon la manifestation d'une « littérature blog » (Taupenot 2017:21), puisque l'auteur garde toujours une proximité importante avec la littérature - déjà comme lecteur, mais aussi en tant qu'écrivain -, il me semble, d'un autre côté, que cette désignation passe à côté du travail constant de reformulation, voire de "remédiation » (Rajewsky 2005) auquel s'adonne François Bon, qui ne se contente pas de passer d'un support à l'autre, mais qui repense et refait les formes en fonction de ces passages entre différents systèmes et différentes plateformes de communication.

La note d'auteur que l'on trouve sur tierslivre.net à propos justement de Tumulte, est très symptomatique de cet investissement continu dans la révision de ces projets multimodales, aussi bien d'écriture que de lecture :

Note du 17 avril 2006 :

On trouvera ci-dessous l'histoire des différentes conceptions du Tumulte. Au terme de cette première année, les éditions Fayard ont accepté de publier les fictions mises en 
ligne au quotidien. Les textes concernés ne sont plus visibles dans la partie publique du site, mais nous travaillons à une forme qui en permette l'accès et la consultation à partir du livre. L'expérience continue avec de nouvelles directions. ${ }^{7}$

Nous pouvons donc affirmer que, chez François Bon, il n'existe pas de confusion, par ailleurs assez commune, entre littérature numérique et littérature numérisée, car celle-ci ne signifie qu'un changement de support de lecture. Son Tiers Livre, par contre, est conçu comme un vrai atelier d'écriture, dont la performance littéraire est radicalement liée au numérique, ce qui signifie que le site fonctionne en soi comme moteur de création d'un macro-texte intégrant mots, son, images et vidéos, et que l'auteur travaille comme éditeur de leurs potentialités interactives à travers des codes informatiques, des algorithmes, et différents liens, à l'image de l'épanchement du rhizome deleuzien.

«L'enjeu c'est le Web, et le nouveau livre, c'est le site. Le site comme livre »notait François Bon il y a dix ans (Bon 2011 : 169-170); l'on pourrait peut-être ajouter comme un archi-livre, un vrai avatar du rêve mallarméen de Livre absolu, même si le côté visionnaire de Bon choisit plutôt une métaphore organique puisée dans la nature :

Nous serions alors chacun l'écrivain d'un seul livre. Ce livre grandirait avec nous, il serait comme un arbre. Il serait fait de toutes nos traces, porterait à jamais toutes les cicatrices et les coupures. De cet arbre, nous saurions définir ce qui est tronc, par lequel nous grandissons, et ce qui est branche, nos chantiers, nos recherches, nos mises en attente, et jusqu'à ce qui est feuillage, où nous frôlons et nous mêlons aux arbres voisins, ce qui serait, par commentaires et réseaux, la limite bruissante de l'arbre personnel. (Bon 2011 : 199-200)

Bien qu'ayant fondé en 2008 la première plateforme d'édition numérique de littérature contemporaine en France - publie.net - ce n'est pas le support de publication en soi qui l'intéresse. Pour l'auteur de Limite (1985), l'édition du livre, qu'il soit imprimé ou numérisé, si elle est conçue comme simple objet mercantile, ou comme fuite en avant des différents agents du secteur, elle ne fait que contribuer à une sorte de "chronique de la mort annoncée », pour rappeler ici le titre devenu célèbre de Gabriel Garcia Marquez. Il a fini par se dissocier de cette plateforme, qui n'aura représenté pour lui qu'un jalon de plus en vue d'inclusion d'une pensée numérique de l'écrit dans l'ensemble de la démarche littéraire. ${ }^{8}$ Dès lors, toute pratique sémiotique, toute expérience que l'auteur met en œuvre (lectures, voyages, photographies, vidéos, ateliers d'écriture, interventions en ligne) constitue déjà le livre à venir. Sa version finale, éventuellement imprimée, puisque l'économie de la vie littéraire et culturelle l'exigent encore, n'en est finalement qu'une de des multiples manifesta- 
tions d'écriture, non plus soumise ni à des frontières insurmontables entre discours ou pratiques artistiques, ni à une temporalité étanche d'exécution, soit encore à une forme de lecture linéaire.

Le lancement officiel, en 2016, de Tiers Livre Éditeur, lié au site homonyme et à une chaîne de vidéo sur Youtube, correspond à l'état actuel d'un projet que François Bon n’hésite pas à considérer comme "renaissance du livre imprimé », à la suite de la diffusion de proximité de l'impression à la demande qui, du même coup, affranchit aussi l'auteur des conditions précaires, voire indignes, qui lui sont de plus en plus imposées par la généralité des maisons d'édition. Maintenant l'auteur est celui qui contrôle de près toutes les étapes de son travail créatif, et il n'est plus forcé de renoncer à aucun aspect des modalités de son écriture, à cause de contraintes éditoriales ou d'exigences commerciales. Il n'a pas, notamment, à abdiquer des expérimentations personnelles, ou de ses lectures-performances convoquant des auteurs et des domaines assez éloignés, du moins au départ, comme c'est le cas de Rabelais, Proust, Rolling Stones ou Led Zeppelin... Il lui est possible, en plus, d’intégrer dans sa démarche de littérature numérique ses projets de traduction, en proposant notamment des éditions bilingues, comme c'est le cas pour les œuvres de Lovecraft et, finalement, de reprendre - en les relisant du point de vue numérique - quelques-uns de ses travaux antérieurs. Gilles Bonnet a parfaitement saisi l'enjeu créatif, et très stimulant pour les lecteurs, de cette « remédiatisation », quand il a noté à propos de l'« écranvain en-son-site »:

Relisant ses propres livres, republiés, augmentés et commentés, Bon en fait des textes d'alluvions, dépositaire de traces qu'il appartient désormais au lecteur de saisir. Démarche archéologique, le paratexte génétique fait scintiller différemment le texte originel, doublant la temporalité horizontale de ma lecture d'une temporalité verticale issue de ce carottage dans les circonstances de la rédaction (Bonnet $2015: \mathrm{s} / \mathrm{p}$ )

Il ne faut pas en déduire que le lecteur idéal des écritures numériques de François Bon sera un chercheur académique dans une fouille de sources, de palimpsestes ou de brouillons, afin d'expliquer en quelque sorte le texte final fixe, qui, finalement, n'existe pas... Au contraire, l'enjeu est de convoquer des lecteurs attirés par le futur des textes en mutation constante.

Le site de François Bon comme interface de lecture se présente, donc, comme un espace ouvert de possibilités, croisant le geste auctorial d'aménagement de rubriques et d'autoréflexion continue du processus d'écriture(s), et le geste du lecteur qui pourra choisir le sens de sa navigation sur la plateforme, ${ }^{9}$ les lignes de fuite qui lui sont proposées par ce cadre d'énonciation numérique. Le lecteur d'une telle instance tierce d'écriture a affaire à la figure d'un auteur qui n'est pas seulement un auteur multiple, créant avec plusieurs discours et intervenant sur plusieurs réseaux 
mais qui est en même un acteur assumé, un performeur acharné de ses propres textes et de ceux des autres; en somme le personnage métalittéraire d'un auteur diffracté «en de multiples micro-identités sur divers supports et plateformes » (Bonnet 2017 : 95), conçues comme des pages d'un dispositif narratif continu.

Pour cet auteur qui ne se soucie plus de savoir s'il continue d'être un écrivain ou pas, car seule l'énergie des différentes écritures l'intéresse, il existe une totale indissociabilité entre lire et écrire, ainsi qu'une force créatrice dans le fait de déclencher des "vases communicants", d'où sa prédisposition à participer activement à une communauté littéraire numérique, à travers le blogroll, avec les adresses de plusieurs blogs de littérature, photographie, musique, etc., que l'auteur côtoie régulièrement. C'est pourquoi l'internaute qui fréquente le Tiers Livre de François Bon peut prendre directement contact avec un ensemble informel et variable d'auteurs et de lecteurs qui assure l'échange d'une pratique, ou qui ont un goût commun de textes disruptifs et inclassables, résultat d'une écriture en ligne qui déploie des systèmes transmédias, et rompt avec des frontières artistiques.

Finalement, je crois que c'est par l'empreinte de cette forme de communication directe, plutôt volontariste, promue par le format blog que l'on entre à fond dans la question du plaisir, car à la question de savoir s'il est toujours possible de parler du fameux plaisir du texte barthésien à l'ère du numérique, force est de répondre qu'une démarche créative comme celle de François Bon le justifie plus que jamais. En fait, aussi bien l'auteur que le lecteur en ligne se trouvent devant un espace de séduction permanente, de la mise en scène d'une « apparition-disparition » (Barthes 1973:19), du fait des liens exposés, des domaines cachés, des envies ou curiosités suscitées chez le lecteur, le tout créant une dialectique constante entre visible et invisible, entre le flux et le fixe. Par ailleurs, tel que Roland Barthes l'avait conçu, le texte littéraire numérique est en lui-même atopique (idem : 69), tout comme sa jouissance (idem : 106), l'un et l'autre manifestant l'impossibilité de vivre hors du texte infini (idem : 77), souvent accompagné du grain de la voix (idem: 115 ), ce que le sémiologue appelait de ses vœux juste à la fin de son Plaisir du Texte (idem : II6).

L'internaute, lecteur-spectateur du Tiers Livre, non seulement compte sur le plaisir dans ce laboratoire d'écriture multimodale et hypertextuelle, mais aussi sur celui d'y participer avec des commentaires (et que François Bon tient explicitement à considérer) $i^{10}$ ce qui élargit le potentiel du versant collectif de cet imaginaire numérique. Le plaisir n'exclut pourtant pas l'exigence, puisque le régime esthétique du mélange des discours artistiques, la pratique de la disruption, ainsi que le tourbillon de références et de renvois pousseront le lecteur à prendre plaisir au « feuilleté de la signifiance » (Barthes 1973:23), à en faire " un véritable acteur de sa lecture » (Bonnet $2017: 17$ ).

En 1920, l'écrivain allemand naturalisé suisse, Hermann Hesse, énumérait trois types de lecteurs, en soulignant que chacun de nous y participe selon les occasions : 
le lecteur naïf, qui ne fait que suivre le livre, qui évalue tout avec «le mètre d'une esthétique » parce qu'il poursuit une convention; le lecteur envisageant la lecture à la façon d'un divertissement, et qui cherche à trouver les failles de l'auteur, et, finalement, le lecteur qui assume une attitude très personnelle, très libre par rapport à ce qu'il est en train de lire, car pour lui le livre n'est qu'un stimulus, un point de départ (Hesse 2018:80-88). Pour ce dernier, le livre serait surtout une construction mentale avant et après l'ensemble de feuilles imprimées. Un siècle plus tard, et malgré un contexte littéraire, social et politique très différent de celui dont témoignait à l'époque l'auteur d'Une bibliothèque idéale," il me semble intéressant de revenir sur cette typologie, car elle continue de fonctionner aussi pour la littérature numérique, et ses différents lecteurs-internautes. En effet, existent toujours ce lecteur qui n'est sensible qu'à ce qu'il reconnaît d'avance comme littéraire, et qu'il associe surtout à quelques aspects conventionnels, ainsi que le lecteur qui appréhende le texte numérique comme un jeu de multiples signes et défis. Pourtant, c'est au niveau du troisième lecteur - pour qui le plaisir de lire est au-delà même du livre, et qui implique une forme de redécouverte de soi et du monde -, que l'on doit continuer à se poser des questions sur les formes de réception et de lecture des textes littéraires à l'ère numérique, non seulement de ceux qui sont déjà écrits sur la Toile, mais aussi des autres, de ceux qui ont été écrits sur d'autres supports, surtout sur papier, et qui n’attendent qu'à redevenir des objets de désir : «Qui donc un jour aurait une solution viable, quand certains pourtant disaient encore le plaisir qu'ils avaient eu au livre, et ce qu'ils y avaient trouvés? »-demande l'auteur de Société des Amis de l'Ancienne Littérature (Bon 2020 : 100), une tradition que François Bon n'est pas près d'ignorer, car son véritable partenaire d'écriture, c'est toute la littérature (Viart 2008 : 156), mais qu'il entend réinvestir tout en faisant route vers le futur.

Moi-même, en essayant d'accompagner, plus ou moins régulièrement, la démarche créatrice du Tiers Livre de François Bon, je me reconnais souvent dans ce troisième lecteur d'Herman Hesse, dans la mesure où j'y navigue, mais pour m'en échapper, c'est-à-dire, pour imaginer et penser après lui, après les questions qu'il me suscite et qui, en général, tournent justement autour des enjeux posés par cette circulation entre deux paradigmes littéraires, l'un plutôt marqué par le format livre en papier et par le champ littéraire tel qu'il a fonctionné surtout à partir du XIXème siècle, et l'autre, construit (aussi) selon des codes informatiques, misant sur la transmédialité et sur la communication directe en réseau à potentiel global. J'insiste sur l'idée de circulation et non pas de simple passage de l'un à l'autre, parce qu'il est tout à fait impossible de jeter à terre plusieurs siècles de tradition, et que nous continuons de vivre à cheval entre ces deux régimes. En guise de conclusion sur ce qui, en rigueur, ne peut être conclu parce qu'il s'agit ici d'un processus toujours en cours, je me permettrai d'énoncer une des questions, à mon avis, la plus déterminante pour que le plaisir de lire-écrire-lire des textes littéraires à l'époque numérique signifie 
effectivement une jouissance, au sens à nouveau barthésien, de bascule ou d'ébranlement (Barthes 1973: 17) du lecteur face aux mots et au monde(s) qu'ils convoquent. Ainsi, comment gérer le risque d'un égarement aliénant du lecteur, du fait de la voracité non pas forcée, certes, mais quand même induite par la navigation sur une plateforme débordante de signes, et en mutation permanente? Autrement dit : comment préserver l'oscillation entre l'immersion dans le flux de l'œuvre hypermédiatique et le recueillement dans la coalescence silencieuse de l'écriture et de la lecture? Chercher des réponses à cet enjeu exige, certes, un investissement dans ce que l'on appelle " humanités numériques », comme champ transdisciplinaire encore assez imprécis. Mais, il ne faudra pas oublier qu'en amont et en aval, cela touche aussi le cœur même de la « recherche d'un nouveau monde » (Bon 2020 : 15-53), avec tout ce qui la présuppose et qui ne saura se faire sans la force poétique des fictions, comme celle de François Bon, qui rêvent du futur comme revisitation d'un passé imparfait.

\section{Notes}

*Ana Paula Coutinho est Professeure habilitée à diriger des recherches à l'Université de Porto (Département d’Études Portugaises et Romanes de la Faculté de Lettres), où elle enseigne surtout la littérature comparée et les études françaises. Docteure en Littérature Comparée (1998) et HDR en Littératures et Cultures Romanes (20I0), elle a toujours travaillé sur la littérature contemporaine dans une perspective comparatiste. Depuis quelques années, elle s'intéresse tout particulièrement à la recherche dans les domaines des interculturalités et des représentations littéraires et artistiques de la migration et de l'exil. Elle a intégré à plusieurs reprises la Direction de l'Instituto de Literatura Comparada et elle actuellement sa coordinatrice scientifique. Elle est aussi la coordinatrice de l'encyclopédie digitale Ulyssei@s. Membre collaborateur du CRIMIC (Université Paris IV), elle a collaboré aussi avec le Programme Non-Lieux de l'Exil (Collège d'Études Mondiales - FMSH). Elle est vice-présidente de l'Alliance Française de Porto. Elle a plusieurs études publiées dans des revues et des ouvrages collectifs, nationaux et étrangers (Colóquio/Letras, Cadernos de Literatura Comparada, Revue de Littérature Comparée, Diogène, Gávea-Brown, Carnets, Latitudes: Cahiers Lusophones, Intercâmbio...). Parmi les livres publiés ou édités: António Ramos Rosa. Mediação Crítica e Criação Poética (Quasi Edições, 2003, Prix Essai Pen-Club) ; Lentes Bifocais - Representações literárias da Diáspora Portuguesa (Afrontamento, 2009), Passages et Naufrages migrants. Les fictions du détroit (avec Maria de Fátima Outeirinho et José Domingues de Almeida), Paris, Harmattan, 2012 ; Nos \& leurs Afriques. Images identitaires et regards croisés. Constructions littéraires fictionnelles des identités africaines cinquante ans après les décolonisations (avec Maria de Fátima Outeirinho et José Domingues de Almeida), Frankfurt, Berlin, 
Peter Lang, 2013 ; Marguerite Duras. Palavras e Imagens da Insistência, ILCML, Colecção Libretos, 2015.

' https://richreading.ppke.hu/ .

${ }^{2}$ Devenue, entre-temps, une des premières revues littéraires francophones uniquement numérique.

${ }^{3}$ Cf. http://remue.net/qui-sommes-nous [ consulté le 20 juin 2020].

${ }^{4}$ S’il n'est pas le seul, il est sûrement un des rares écrivains contemporains qui pouvait exposer, il y a déjà une dizaine d'années, une « autobiographie numérique » au micro de France Culture. [En ligne ]: https:// www.franceculture.fr/emissions/place-de-la-toile/francois-bon-autobiographie-numerique.

${ }^{5}$ Voir, par exemple, son intervention à l'occasion du colloque international « Vers une littérature mondiale à l'heure du numérique? », intitulée « Qu'est-ce que le Web change à l'auteur de littérature? » (Bon 2015).

${ }^{6}$ https://www.dictionnaire-academie.fr/article/A9LIO74.

${ }^{7}$ https://www.tierslivre.net/2tumulte/spip.php?articlel.

${ }^{8}$ Voir - Manifeste pour une pensée numérique de l'écrit. https://www.youtube.com/watch?v=8kFnttılo9o.

9 De noter que le tierslivre.net comprend un « espace abonnés ». En gérant différents niveaux du contenu du site, l'auteur appelle aussi à différents degrés de lecture de son Livre numérique.

${ }^{10}$ Voir sa réflexion, assez stimulante et innovatrice à plusieurs titres : « (écrire) que les commentaires ne sont pas une écriture du bas » ( Bon 2011 : 255-261)

"Titre de la traduction française. J'ai pourtant utilisé la traduction portugaise.

\section{Bibliographie}

Barthes, Roland (1973), Le plaisir du texte. Paris, Éditions du Seuil. Bon, François (1985), Limite. Paris, Minuit.

-- (2011), Après le livre. Paris, Éditions du Seuil.

-- (2012), Autobiographies des objets. Paris, Éditions du Seuil.

-- (2015), Tiers Livre, "Qu'est-ce que le Web change à l'auteur de littérature?", [En ligne]. mis à jour le 19 avril 2016. http://www.tierslivre.net/spip/spip. php?article4224 (Consulté le 18 juillet 2020).

-- (2020), Société des Amis de l'Ancienne Littérature précédé de Recherche d'un nouveau monde. Tiers Livre éditeur.

Bonnet, Gilles (2012), François Bon, d'un monde en bascule. Chêne-Bourg, La Baconnière.

-- (2015), « On relit toujours avec de soi: l'ecranvain en son site », Komodo 2l, I. En ligne: http://komodo2I.fr/on-relit-toujours-avec-de-soi-lecranvain-en-son-site/ [consulté le 28 octobre 2020]. 
-- (2017), Pour une littérature numérique. Littérature et internet. Paris, Éditions Hermann.

Hesse, Hermann (2018), Uma Biblioteca da Literatura Universal. Lisboa, Cavalo de Ferro.

Rajewsky, Irina O. (2005), « Intermediality, intertextuality, and remediation. A Literary Perspective on Intermediality », Intermédialités, 6, pp.43-64.

Taupenot, Maé (2017), Étude du Tiers Livre de François Bon de 2005 à aujourd'hui, une renégociation des pratiques d'écriture par le numérique, Université Lyon 2. Consulté dans https://www.enssib.fr/bibliotheque-numerique/ documents/67862-etude-du-tiers-livre-de-francois-bon-de-2005-a-aujourd-huiune-renegociation-des-pratiques-d-ecriture-par-le-numerique.pdf [Consulté le 30 juillet 2020].

Viart, Dominique (2008), François Bon. Étude de l'œeuvre. Paris, Bordas. 